\title{
Les acquisitions de terres rurales par les « cadres» en Côte d'Ivoire : premiers enseignements
}

\author{
Honneo Gabin Tarrouth ${ }^{1, *}$ et Jean-Philippe Colin ${ }^{2}$ \\ ${ }^{1}$ Université Felix Houphouët Boigny, 04 BP 2450, 00213 Abidjan, Côte d'Ivoire \\ 2 IRD, UMR GRED/GIS Pôle foncier, 911 avenue Agropolis, BP 64501, 34394 Montpellier Cedex 5, France
}

\begin{abstract}
Résumé - En Afrique, la question foncière est actuellement dominée par le thème des acquisitions de terre à grande échelle, mais la place des élites nationales dans cette dynamique reste peu traitée. Ce texte présente les premiers résultats d'une recherche sur les acquisitions « marchandes » de terres par les « cadres » en Côte d'Ivoire. Il montre que l'achat pour la réalisation de plantations d'hévéa domine et que les superficies concernées restent relativement limitées. Comme dans le cas de transactions entre villageois (généralement entre autochtones et migrants), les acquisitions foncières impliquant des cadres restent marquées par un enchâssement social. Ces premiers résultats conduisent à relativiser la spécificité des transferts fonciers au bénéfice des cadres par rapport aux transferts n'impliquant que des villageois.
\end{abstract}

Mots clés : élites / marché foncier / transaction foncière / sécurisation des transactions / hévéa

\begin{abstract}
Farmland acquisition by national elites in Côte d'Ivoire: first insights. In Africa, the issue of land grabbing currently dominates debates about land management, but the role of national elites in this process is rarely empirically addressed. This text aims at offering a first analysis of land market acquisitions by the national elite ("cadres") in Côte d'Ivoire. It shows that land purchase for rubber planting predominates, and that the areas concerned by the acquisitions remain relatively modest. As in the case of transactions among villagers (mostly between natives and migrants), those involving cadres are socially embedded. Those first results question the specificity of land transfers benefitting cadres, compared to transfers involving villagers.
\end{abstract}

Keywords: national elite / land market / land transaction / securing land transactions / rubber

\section{Introduction}

En Afrique, la question foncière est dominée depuis quelques années, en termes médiatiques, politiques et de publications scientifiques, par le thème des investissements fonciers à grande échelle. L'attention se porte le plus souvent sur les investisseurs étrangers, mais la place des élites nationales dans cette dynamique est aussi de plus en plus reconnue. Ce texte contribue à la réflexion dans ce champ à travers l'analyse des acquisitions marchandes de terres par les «cadres » en Côte d'Ivoire. Par «cadres ", on entend, dans le contexte rural ivoirien, tout individu développant une activité professionnelle en milieu urbain et doté d'un capital culturel, social, économique ou politique jugé important par la population rurale. Au-delà de cette acception très large, la caractéristique clé qui nous intéresse ici est le statut professionnel « non rural » des acquéreurs, quelle que soit la superficie acquise. Il s'agit de cadres extérieurs à la communauté villageoise, qui y acquièrent des terres, les transactions marchandes intra-communautaires restant absentes ou très marginales.

\footnotetext{
*Auteur de correspondance. gabintarrouth@yahoo.fr
}

Une première section du texte présente le questionnement et les conditions de production des données de la recherche. La deuxième section propose une caractérisation des acteurs et leurs motivations. La dernière section traite de la caractérisation des transactions.

\section{Questionnement et méthode}

L'importance des nationaux (individus ou entreprises) dans la dynamique des « grandes acquisitions » est largement sous-estimée dans les débats (Cotula, 2012). La littérature récente relative à l'Afrique de l'Ouest francophone mentionne l'acquisition de terres par les élites nationales au Sénégal (Faye et al., 2011), au Bénin (Angsthelm et al., 2010) ou au Burkina Faso (GRAF, 2011 ; Mathieu et al., 2003 ; Ouédraogo, 2003). Une autre étude récente traite des acquisitions de terres par des nationaux au Bénin, au Burkina Faso et au Niger (Hilhorst et al., 2011). Ces acquisitions portent, sauf exceptions, sur des superficies beaucoup moins importantes (de quelques hectares à quelques centaines d'hectares) que celles avancées dans la littérature sur les «grandes acquisitions » (souvent plus de $1000 \mathrm{ha})$. 
La question des acquisitions foncières par les élites nationales revêt une importance toute particulière en Côte d'Ivoire, avec l'engouement marqué, ces dernières années, des cadres pour la création de plantations pérennes - hévéa en tout premier lieu. La présence des élites nationales dans l'agriculture n'est pas un fait nouveau. L'État, par le passé, a largement concédé à de « grands cadres » des terres situées dans des forêts classées. Ceux d'entre eux qui étaient originaires de la zone forestière avaient également accès à la terre dans leur village d'origine pour y créer des plantations pérennes, à l'image du président Houphouët Boigny. L'implication de ce type d'acteurs a été bien mise en évidence dans l'histoire agraire de la Côte d'Ivoire (Affou Yapi, 1987). La dynamique engagée actuellement est différente, du fait du recours au marché foncier pour accéder à la terre et de l'intensité plus forte du phénomène. Depuis le début et surtout le milieu des années 2000, des acquisitions de terres en zone forestière ou en zone de contact forêt/savane, par des cadres, pour la réalisation de plantations d'hévéa, sont évoquées dans la littérature, mais très peu d'études leur ont été consacrées (voir Ruf, 2011, 2013).

Ce texte présente les premiers résultats d'une recherche doctorale en cours, visant à identifier et discuter les déterminants, les conditions et les conséquences des acquisitions de terres rurales par les cadres en Côte d'Ivoire. L'article se concentre sur la caractérisation des acteurs et des transactions : quels sont les acteurs de ces transactions - audelà du seul qualificatif de «cadres », s'agissant des acquéreurs ? Comment s'opère la rencontre des acteurs, quel est le rôle joué par le capital social, par des intermédiaires ? Quel est le contenu des transactions, ces transactions sont-elles complètes, sont-elles enchâssées socialement ?

Pour les enquêtes de terrain, deux sites où les cadres sont actifs en matière d'acquisitions, foncières, ont été retenus : Fresco/Grand-Lahou, sur la côte à l'ouest d'Abidjan, et Toumodi, dans le centre, qui est une zone de contact forêtsavane. Ont été enquêtés 14 acquéreurs et 20 cédants (dont 8 « paires ») sur Fresco/Grand Lahou, et cinq acquéreurs et 19 cédants sur Toumodi. Nous avons également réalisé des entretiens avec les sous-préfets, les directeurs départementaux de l'agriculture, les autorités villageoises, les associations de jeunes, des démarcheurs ruraux (terme local utilisé par les enquêtés). Nous avons par ailleurs réalisé des enquêtes (38) à Abidjan auprès de cadres acquéreurs qui résident dans cette ville (quelle que soit la localisation des parcelles acquises). En définitive, l'analyse qui suit repose sur des enquêtes conduites auprès de 57 acquéreurs et 36 cédants individuels, avec en sus trois études de cas sur des cessions réalisées par des villages. Les données relatives aux cédants et aux acquéreurs sont directement issues des enquêtes effectuées auprès de ceux-ci, mais certaines informations ont aussi pu être collectées indirectement, en étant fournies par la partie à la transaction enquêtée, en portant sur la partie non enquêtée. Il n'a en effet été possible que dans 8 cas d'enquêter les deux parties d'une même transaction (« paire »).

\section{Caractérisation des acteurs et motivations}

Les cédants sont en majorité autochtones (88 sur 93). Ce sont des chefs de famille (51), des jeunes, au sens de cadets sociaux (37), des chefs de terre (2), des collectifs villageois (3). Trois des cédants sont des femmes (toutes chefs de famille).

Les raisons invoquées par les cédants pour justifier les transactions sont diverses : en premier lieu, afin d'avoir les moyens de construire une maison décente, mais aussi pour l'achat de moto ou de voiture. Les trois cessions réalisées par les « collectifs villageois » visaient, elles, à permettre, en contrepartie des cessions, la réalisation par de grands cadres acquéreurs, d'infrastructures pour le développement du village. Les «ventes de détresse », souvent considérées comme un facteur majeur des cessions foncières, ne semblent pas avoir revêtu une importance notable dans le cas des transactions étudiées.

Le niveau d'instruction des cadres est élevé : 47 sur 57 ont un niveau d'éducation supérieure (variable documentée uniquement auprès des acquéreurs). On peut distinguer les acquéreurs sur la base d'une typologie professionnelle simple (variable documentée pour les 93 acquéreurs identifiés directement ou indirectement) (Tab. 1). Par « petits cadres », nous entendons des personnes ayant un rôle d'exécutant dans une organisation (entreprise, administration) : sous-officier, instituteur, etc. ; « cadres moyens » renvoie à des professions

Tableau 1. Typologie des cadres de notre échantillon.

Table 1. Cadres: a typology.

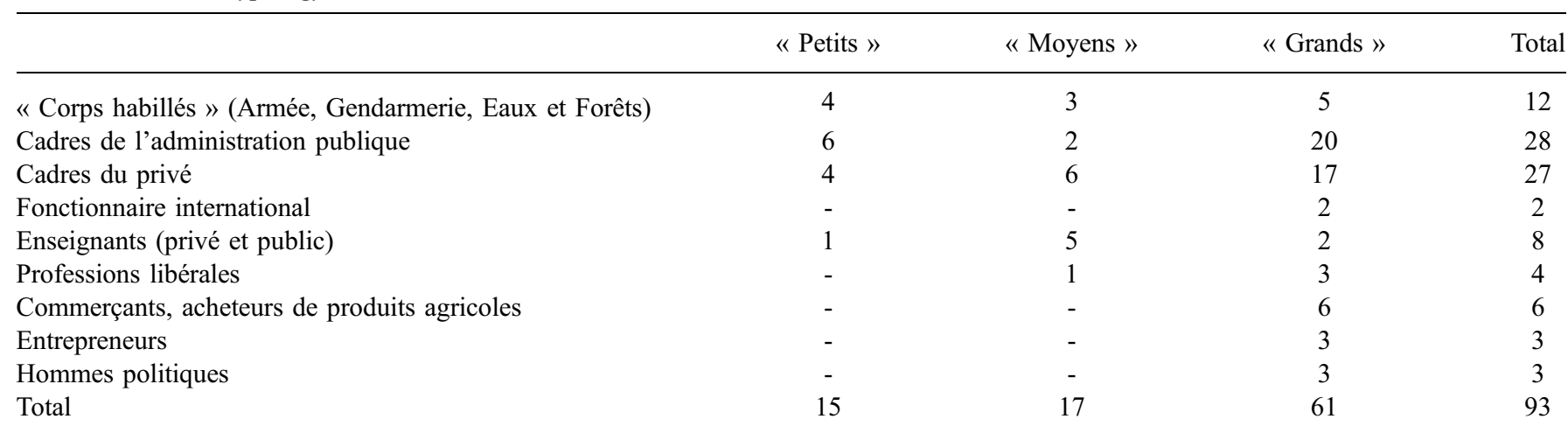

Source : nos enquêtes. 
intermédiaires : officiers subalternes, professeurs de l'enseignement secondaire, etc. ; les « grands cadres » occupent une position importante dans la hiérarchie d'une organisation (officier supérieur, directeur ou sous-directeur d'administration publique ou d'entreprise privée, chef comptable, etc.), exercent une profession libérale (médecin, avocat), sont entrepreneurs, grands commerçants, ou encore hommes politiques.

Le choix des localités où les cadres acquièrent des terres s'explique par leur relative proximité d'Abidjan (lieu de résidence habituelle des cadres) ou leur accessibilité en termes d'infrastructure routière, et par leur réputation de régions où il reste encore de la forêt ou des jachères disponibles.

L'acquisition foncière vise avant tout à permettre la réalisation d'une plantation d'hévéa, qui offre trois intérêts majeurs qui sont à mettre en rapport avec les caractéristiques de cette culture : il s'agit d'une culture pérenne (production sur le long terme, avec des frais d'exploitation réduits une fois la plantation réalisée), qui assure des revenus réguliers au long de l'année, et avec un retour sur investissement à long terme vu comme sûr.

\section{Caractérisation des transactions}

\subsection{Types de transaction et superficies}

Trois modalités d'accès à la terre ont été identifiées.

Premièrement, l'achat. Le prix d'achat de la terre varie entre 100000 et $200000 \mathrm{FCFA} /$ ha en francs courants, avec des valeurs moyennes de $100000 \mathrm{FCFA} /$ ha entre 2004 et 2007 , de l'ordre de $140000 \mathrm{FCFA} / \mathrm{ha}$ entre 2008 et 2010, et 200000 FCFA/ha pour les transactions plus récentes $(1000 \mathrm{FCFA}=$ $1,52 €)$. Les variations s'expliquent par la nature de la végétation, les relations entre le cédant et l'acquéreur, le besoin financier plus ou moins urgent du cédant et les disponibilités monétaires de l'acquéreur.

Trois des ventes ont été réalisées par des «collectifs villageois ». Dans le premier cas, il s'agissait de 67 ha de terres collectives du village, correspondant à une savane inexploitée, avec en contrepartie un projet d'électrification et de construction d'un château d'eau, et un versement additionnel de $50000 \mathrm{FCFA} / \mathrm{ha}$. La somme reçue a été utilisée par le chef du village pour la création de deux puits traditionnels dans le village. Dans le deuxième cas, il s'agissait de la vente de 180 ha « en brousse », non exploités, réalisée au nom du village mais touchant des terres contrôlées par une famille fondatrice du village, détentrice d'un patrimoine important. Cette cession à un grand homme politique a été effectuée contre l'engagement de réaliser l'électrification du village et l'installation d'un château d'eau, avec en sus le versement de $50000 \mathrm{FCFA} / \mathrm{ha}$ bénéficiant à la famille cédante, ainsi que la réalisation d'une plantation d'hévéa de 10 ha pour le chef de famille. Le troisième cas correspond à la vente de 40 ha de bas-fonds inexploités de statut collectif villageois (100 $000 \mathrm{FCFA} / \mathrm{ha})$. La somme perçue a été utilisée par la chefferie villageoise pour le reprofilage d'une piste menant à la ville de Grand-Lahou et l'achèvement d'un château d'eau collectif.

Deuxièmement, le Planter-Partager (P\&P) est un type de contrat qui peut être défini en termes génériques comme un arrangement par lequel un exploitant gagne l'accès à un droit d'usage à long terme, voire à un droit de propriété du sol, en mettant en valeur une terre par la réalisation d'une plantation pérenne, et en rétrocédant au propriétaire foncier, lorsque la plantation entre en production, une partie de la plantation créée ou de sa production. Différents types de contrats de P\&P peuvent être distingués en Côte d'Ivoire, selon l'objet du partage : la plantation uniquement, la plantation et la terre qui la porte, ou la production (Colin et Ruf, 2011). Un seul type de contrat de P\&P impliquant des cadres a été identifié lors de nos enquêtes. Il s'agit du contrat avec partage de la plantation à l'entrée en production, le cédant conservant son droit de propriété sur l'ensemble de la terre. Le partage est réalisé à la moitié ( 2 parcelles) ou au tiers $(1 / 3$ pour le cédant, 13 parcelles). Le preneur verse parfois une somme fixe au moment de l'accord (20 000 à $40000 \mathrm{FCFA} / \mathrm{ha}$ ).

Troisièmement, nous avons identifié deux cas d'accès à un droit d'usage à long terme avec paiement intégral ex ante de la rente. Dans un cas, un montant de $110000 \mathrm{FCFA} /$ ha est versé en trois fois (sur un an) pour 22 ha engagés pour 70 ans. Dans l'autre cas, $115000 \mathrm{FCFA} /$ ha sont versés en deux fois (sur une année) pour $30 \mathrm{ha}$, avec engagement sur 50 ans, outre la fourniture d'un bœuf, d'un casier de vin, d'une bouteille de gin et de 20 litres de vin de palme.

L'achat domine largement, avec 82 transactions et 2125 ha, contre 15 transactions et 329 ha pour le $\mathrm{P} \& \mathrm{P}$, et deux transactions et 52 ha pour le droit d'usage à long terme.

Tous types de transactions confondus, la superficie acquise par cadre varie de 3 à 247 ha pour les achats, de 9 à 70 ha (avant partage) pour les contrats de $\mathrm{P} \& \mathrm{P}$, et de 22 à 30 ha pour les deux cas d'acquisition du droit d'usage à long terme avec paiement intégral ex ante. On note quelques cumuls d'achats ou de prise en P\&P par un même acquéreur (5 cas) et de cessions de plusieurs parcelles par un même acteur ( 5 cas également).

La superficie moyenne acquise par type de cadres (superficie totale en cas de cumul) augmente des «petits cadres » aux « cadres moyens » et aux « grands cadres » (Tab. 2). Il n'y a cependant pas de relation systématique entre les superficies acquises et le type de cadre : tous les « grands cadres » ne sont pas de grands acquéreurs, et à l'inverse on trouve parmi ces derniers des cadres qualifiés de « petits » ou « moyens ».

La première acquisition identifiée dans nos enquêtes a été réalisée en $2004 ; 63 \%$ des acquisitions ont été réalisées entre 2004 et 2010, $37 \%$ depuis 2010. Lors de la transaction, les parcelles acquises étaient essentiellement en jachère (774 ha) ou occupées par des plantations abandonnées ou des friches (données relatives aux 57 cadres enquêtés). Lors des enquêtes, 601 ha avaient été plantés en hévéa depuis l'acquisition, 142 ha étaient en cours de plantation, 30 étaient en cultures vivrières. Les acquéreurs projetaient de planter essentiellement de l'hévéa (253 ha) sur les superficies restant à mettre en valeur (outre 42 ha de palmier et 40 ha de cultures vivrières). Le fait que cette mise en valeur n'ait pas encore été totale lors des enquêtes était justifié par les enquêtés par le caractère récent de l'acquisition (4 cas), l'importance de la superficie (4 cas), un manque de moyens $(3 \mathrm{cas})$ et un conflit sur la parcelle $(3 \mathrm{cas})$.

\subsection{Enchâssement social des transactions et droits transférés}

L'enchâssement social du marché, dans l'acception retenue par Granovetter (1985), vise à rendre compte de l'insertion des actions économiques dans des réseaux sociaux. Une deuxième 
Tableau 2. Superficie acquise selon les types de cadres.

Table 2. Area acquired according to the types of cadres.

\begin{tabular}{|c|c|c|c|c|c|}
\hline & \multicolumn{2}{|c|}{ Nombre d'acquéreurs } & \multicolumn{2}{|c|}{ Superficie totale acquise (ha) } & \multirow{2}{*}{$\begin{array}{l}\text { Superficie moyenne } \\
\text { par acquéreur (ha) }\end{array}$} \\
\hline & Nombre & $\%$ & Hectares & $\%$ & \\
\hline « Cadres moyens» & 17 & 18 & 320 & 13 & 19 \\
\hline « Grands cadres » & 61 & 66 & 1951 & 78 & 32 \\
\hline
\end{tabular}

Source : nos enquêtes.

dimension de l'enchâssement, qualifiée de «dimension foncière » par Chauveau et Colin (2010), s'exprime entre les parties à la transaction; elle concerne l'interprétation de la transaction par les parties (et, ultérieurement, leurs héritiers) relativement à l'objet de la transaction (la terre ou le droit d'exploitation), la légitimité du transfert du point de vue du groupe social d'appartenance du cédant, et le caractère libératoire ou pas de la transaction - le fait que la fin de la transaction éteigne ou non toute autre obligation entre les partenaires. Une troisième dimension possible de l'enchâssement des transferts fonciers, "socio-politique ", renvoie à la régulation de l'insertion de «non-locaux » dans les communautés d'accueil, lorsque les transferts fonciers impliquent ces derniers (Chauveau et Colin, 2010). On retrouve dans nos enquêtes ces différentes dimensions de l'enchâssement des transactions.

\subsubsection{Le rôle des réseaux}

Les acquisitions de terres par les cadres sont marquées par un fort enchâssement dans les réseaux sociaux. Pour accéder à la ressource foncière, les acquéreurs, dans leur grande majorité, mobilisent leurs relations (Tab. 3). Une originalité des transactions impliquant des cadres est le rôle joué par les démarcheurs, que l'on ne retrouve pas dans les transferts fonciers concernant d'autres types d'acquéreurs. Nous qualifions de démarcheurs, des intermédiaires qui se font explicitement rémunérer pour leurs services - certains demandent une somme fixe par hectare (24 cas), allant de 5000 à $40000 \mathrm{FCFA} / \mathrm{ha}$, d'autres sont rémunérés au pourcentage du montant total de la transaction ( 2 cas, l'un prenant $10 \%$, l'autre $20 \%$ ). Ces démarcheurs peuvent être d'ores et déjà présents dans les réseaux des cadres, ou être contactés à travers ces réseaux. Un démarcheur apporte sa connaissance des zones où il y a des friches disponibles, connaît les cédants potentiels, établit le contact entre acquéreur et cédant, et est souvent impliqué dans la négociation. À la différence des démarcheurs, les « autres intermédiaires » ne sont pas rémunérés au sens strict du terme : leur intervention relève du service rendu. Certains ont bénéficié en retour d'un geste de reconnaissance non sollicité, en espèce ou en nature, essentiellement symbolique.

\subsubsection{La dimension foncière de l'enchâssement des transactions}

La dimension foncière de l'enchâssement des transactions a été largement décryptée dans le cas des transferts fonciers entre autochtones et planteurs immigrants - les « étrangers » (au sens de non-autochtones), ivoiriens ou non-nationaux (Chauveau et Colin, 2010).

Dans le cas des achats réalisés par des cadres, la transaction porte indiscutablement, pour tous les acquéreurs enquêtés, sur la terre, sur laquelle ils considèrent explicitement détenir l'ensemble du faisceau de droits, sans interférence du cédant : le droit d'exploiter la parcelle achetée à leur convenance, de la vendre, de la céder en faire-valoir indirect, de la mettre en gage, de la transmettre en héritage. L'achat est vu par ces cadres comme les libérant de toute obligation vis-à-vis du cédant et faisant d'eux des propriétaires au sens fort du terme. La seule intervention envisagée du cédant est lors de la future certification foncière, lorsque certains cadres enquêtés (41 acheteurs sur 50) reconnaissent que l'accord du cédant sera nécessaire dans la procédure.

Tableau 3. Réseaux de relations mobilisés lors des transactions.

Table 3. Accessing land through networks.

\begin{tabular}{|c|c|c|c|c|c|c|c|}
\hline & \multicolumn{6}{|c|}{ Réseaux de relations } & \multirow{2}{*}{$\begin{array}{c}\text { Sans } \\
\text { intermédiair }\end{array}$} \\
\hline & Parents & Amis & $\begin{array}{c}\text { Relations } \\
\text { professionnelles }\end{array}$ & Démarcheurs & Autres intermédiaires & Total & \\
\hline Acquéreurs & 7 & 23 & 14 & 8 & 8 & 60 & 3 \\
\hline Cédants & 1 & 9 & - & 18 & 5 & 33 & 3 \\
\hline Total & 8 & 32 & 14 & 26 & 13 & 93 & 6 \\
\hline
\end{tabular}

Source : nos enquêtes. 
Tableau 4. Perception du cédant relativement aux droits de l'acquéreur.

Table 4. Assignor's perception of the cadre's rights.

\begin{tabular}{|c|c|c|c|c|}
\hline & & Vente (40 enquêtés) & P\&P (3 enquêtés) & $\begin{array}{l}\text { Droit d'usage à long terme } \\
\text { ( } 2 \text { enquêtés) }\end{array}$ \\
\hline \multirow{6}{*}{$\begin{array}{l}\text { Droits reconnus } \\
\text { à l'acquéreur } \\
\text { par le cédant }\end{array}$} & Usage sans restriction & 40 oui & $\begin{array}{l}3 \text { non (culture pérenne } \\
\text { spécifiée) }\end{array}$ & 1 oui / 1 non \\
\hline & Vendre & 35 oui / 5 non & 3 oui (plantation) ${ }^{\mathrm{a}}$ & 1 oui / 1 non (plantation) ${ }^{a}$ \\
\hline & Donner & 38 oui / 2 non & 3 oui (plantation) ${ }^{a}$ & 2 oui (plantation) ${ }^{\mathrm{a}}$ \\
\hline & Transmettre en héritage & 39 oui / 1 non & 3 oui (plantation) ${ }^{a}$ & 2 oui (plantation) ${ }^{a}$ \\
\hline & Mettre en garantie & 38 oui / 2 non & 3 oui (plantation) ${ }^{a}$ & 2 oui (plantation) ${ }^{\mathrm{a}}$ \\
\hline & Certifier & 39 oui / 1 non & 3 non & 2 non \\
\hline
\end{tabular}

Source : nos enquêtes.

${ }^{a}$ Droit ne concernant que les arbres plantés et non la terre.

${ }^{\mathrm{b}}$ Pour des cultures vivrières associées à la plantation pérenne les premières années de la plantation.

On ne retrouve pas une vision partagée parmi les cédants. Si la majorité (34 sur 40) affirme que l'acheteur peut exercer tous les droits sur la parcelle, les autres contestent certains droits - droit de vendre la parcelle ou droit de demander un certificat foncier, vus comme la perte définitive de tous leurs droits sur la parcelle cédée - et considèrent que ce n'est pas la terre qui est cédée, mais un droit d'usage.

On note, dans le cas des contrats de P\&P analysés ici, l'existence du même type d'incomplétude que dans les contrats de P\&P noués entre villageois et relevée par Colin (2013), malgré le niveau d'instruction élevé chez les cadres enquêtés : absence de spécification concernant la réalisation de la plantation (intrants utilisés et itinéraire technique, calendrier de réalisation, mode concret de partage), absence d'anticipation d'événements fortuits (comme une destruction accidentelle de la plantation), non-explicitation de critères permettant de déterminer l'échéance du contrat. Cette incomplétude peut être interprétée en termes de rationalité limitée, pour des acteurs qui ne maîtrisent pas la culture de l'hévéa et qui n'ont pas d'expérience quant aux contrats de $\mathrm{P} \& \mathrm{P}$ - dont ils retiennent fondamentalement le simple principe de partage de la plantation. De fait, ce défaut d'anticipation était flagrant lors des entretiens : c'est lorsque nous posions certaines questions relatives aux termes des contrats de $\mathrm{P} \& \mathrm{P}$ que les preneurs réalisaient qu'ils n'avaient pas abordé ces points lors de la transaction.

De façon générale, les relations entre le cédant et l'acquéreur (quel que soit le type de transaction) sont maintenues après la transaction, à travers des aides apportées par les acquéreurs au cédant ou à sa famille, après sollicitation ou non (72 transactions sur 99). Cette aide peut prendre la forme d'un versement d'argent (5000 à 50000 FCFA) en fin d'année, ou pour des funérailles, des frais de santé, etc., ou encore simplement lors de visites au village. Des « gestes de reconnaissance " sont également souvent faits en nature : quelques poulets en fin d'année, un sac de riz, un carton de savon, une bouteille d'alcool, un casier de vin... Ces gestes ne correspondent pas, selon les dires des acquéreurs concernés, à un « devoir », au sens strict du terme, qu'ils auraient vis-à-vis du cédant, mais ils estiment qu'ils lui restent redevables, non sur un registre économique, mais sur ceux de la morale et de la sociabilité. Dans quelques cas, le caractère instrumental du maintien de bonnes relations avec les cédants est explicite, ces attentions étant présentées comme un élément de sécurisation des droits acquis ; la question, fondamentale, de la sécurisation des transactions sera abordée dans un prochain article.

\subsubsection{La dimension socio-politique de l'enchâssement des transactions}

Nos enquêtes font apparaître deux des composantes de la dimension socio-politique de l'enchâssement des transactions : l'aide apportée par des cadres pour la réalisation d'investissements au profit de la communauté villageoise, et leur appui à cette dernière dans ses rapports avec « l'extérieur ». Dans l'un des villages, quatre grands cadres ayant acquis de la terre dans le village ont répondu favorablement après avoir été sollicités par les autorités villageoises pour contribuer à la construction d'un établissement primaire et d'une cantine scolaire. Dans un autre village, un avocat ayant acquis de la terre est devenu le conseiller juridique, à titre gracieux, du village ; il a par ailleurs contribué (après l'acquisition) aux frais de reprofilage d'une piste.

La perception des cédants quant aux droits transférés lors des transactions varie d'un cédant à un autre, mais aussi selon le type de contrat (Tab. 4).

Un enseignement fort de nos enquêtes auprès des cédants est le fait que ces derniers, lorsqu'ils vendent de la terre à des cadres, reconnaissent pour la plupart le transfert de l'intégralité du faisceau de droits, i.e. qu'il s'agit d'une vente complète.

\section{Conclusion}

Trois types de transactions impliquant des cadres ont été identifiés sur notre terrain : l'achat, le Planter-Partager et le transfert d'un droit d'usage à long terme; l'achat domine largement. Les superficies acquises sont très variables, mais restent relativement limitées, comparativement aux constats de la littérature traitant des grandes acquisitions foncières. Les acquisitions par les cadres sont marquées par un fort enchâssement dans des réseaux sociaux préexistants et par le rôle joué par les démarcheurs. Les dimensions « ressource 
foncière » et « socio-politique » de l'enchâssement, évoquées dans la littérature traitant des transactions foncières en milieu villageois, sont également présentes dans le cas des acquisitions réalisées par les cadres. Ces premiers résultats conduisent donc à relativiser la spécificité des transferts fonciers au bénéfice des cadres, par rapport aux autres transferts dans lesquels cette catégorie sociale n'est pas impliquée.

Il reste à voir si on retrouve cette relative absence de spécificité dans la charge conflictuelle des transferts fonciers au bénéfice des cadres, dans les modalités de sécurisation des transactions et dans la productivité de la ressource foncière. Ce sera l'objet d'articles à venir.

\section{Références}

Affou Yapi S. 1987. Les planteurs absentéistes de Côte d'Ivoire, Paris: ORSTOM. http://horizon.documentation.ird.fr/exl-doc/pleins tex tes/pleins_textes_5/pt5/travaux_d/24848.pdf.

Angsthelm B, Mahinou N, Lukacs AM. 2010. Agricultures familiales et sociétés civiles face aux investissements massifs dans les terres agricoles au Bénin. Synergie paysanne, CCFD et Coordination Sud, Cotonou. https://hal-paris1.archives-ouvertes.fr/dumas-00948184/ document.

Chauveau JP, Colin JP. 2010. Customary transfers and land sales in Côte d'Ivoire: revisiting the embeddedness issue. Africa 80: 81-103.

Colin JP. 2013. Securing rural land transactions in Africa. An Ivorian perspective. Land Use Policy 31: 430-440.

Colin JP, Ruf F. 2011. Une économie de plantation en devenir. L'essor des contrats de Planter-Partager comme innovation institutionnelle dans les rapports entre autochtones et étrangers en Côte d'Ivoire. Rev. Tiers Monde 207: 169-187.
Cotula L. 2012. The international political economy of the global land rush: a critical appraisal of trends, scale, geography and drivers. J. Peasant Stud. 39: 649-680.

Faye IM, Benkahla A, Touré O, Seck SM, Ba CO. 2011. Les acquisitions de terres à grande échelle au Sénégal : description d'un nouveau phénomène. Dakar: Initiative Prospective Agricole et Rurale (IPAR). http://www.ipar.sn/sites/default/files/Rapport\% 20ATGE\%20version\%20finale.pdf.

GRAF. 2011. Agrobusiness au Burkina Faso. Quels effets sur le foncier et la modernisation agricole ? Ouadagougou: GRAF. http:// www.graf-bf.org/IMG/pdf/Agrobusiness_au_BFA.pdf.

Granovetter M. 1985. Economic action and social structure: the problem of embeddedness. Am. J. Sociol. 91: 481-510.

Hilhorst T, Nelen J, Traoré N. 2011. Agrarian change below the radar screen: rising farmland acquisitions by domestic investors in West Africa Results from a survey in Benin, Burkina Faso and Niger. Amsterdam: Royal Tropical Institute and SNV Netherlands Development Organisation. http://www.snv.ngo/download/publica tions/agrarian_change_under_radar_screen_kit_snv.pdf.

Mathieu P, Lavigne Delville P, Paré L, Zongo M, Ouédraogo H. 2003. Sécuriser les transactions foncières dans l'ouest du Burkina Faso. Londres: IIED. http://pubs.iied.org/pdfs/9270IIED.pdf.

Ouédraogo M. 2003. Les nouveaux acteurs et la promotion des activités agro-sylvopastorales dans le sud du Burkina Faso : faux départ ou inexpérience ? Londres: IIED. http://pubs.iied.org/pdfs/ 9271IIED.pdf.

Ruf F. 2011. Pareto en Côte d'Ivoire : cycle du caoutchouc et concentration foncière. In: Jul-Larsen E, Laurent PJ, Le Meur PY, Léonard E, eds. Une anthropologie entre pouvoirs et histoire. Conversations autour de l'œuvre de Jean-Pierre Chauveau. Paris: Karthala-IRD-APAD, pp. 59-91.

Ruf F. 2013. Agriculture contractuelle et boom de l'hévéaculture villageoise en Côte d'Ivoire. Cah. Agric. 22: 46-52. http://dx.doi. org/10.1684/agr.2012.0575.

Cite this article as: Tarrouth HG, Colin J-P. 2016. Les acquisitions de terres rurales par les « cadres » en Côte d'Ivoire : premiers enseignements. Cah. Agric. 25: 15005. 